\title{
Wernicke's aphasia reflects a combination of acoustic-phonological and semantic control deficits: A case-series comparison of Wernicke's aphasia, semantic dementia and semantic aphasia
}

\author{
Holly Robson, Karen Sage, Matthew A. Lambon Ralph* \\ Neuroscience and Aphasia Research Unit (NARU), University of Manchester, UK
}

\section{A R T I C L E I N F O}

\section{Article history:}

Received 17 January 2011

Received in revised form

14 November 2011

Accepted 29 November 2011

Available online 8 December 2011

\section{Keywords:}

Wernicke's aphasia

Posterior STG

Posterior MTG

Comprehension

Phonology

Semantics

\begin{abstract}
A B S T R A C T
Wernicke's aphasia (WA) is the classical neurological model of comprehension impairment and, as a result, the posterior temporal lobe is assumed to be critical to semantic cognition. This conclusion is potentially confused by (a) the existence of patient groups with semantic impairment following damage to other brain regions (semantic dementia and semantic aphasia) and (b) an ongoing debate about the underlying causes of comprehension impairment in WA. By directly comparing these three patient groups for the first time, we demonstrate that the comprehension impairment in Wernicke's aphasia is best accounted for by dual deficits in acoustic-phonological analysis (associated with pSTG) and semantic cognition (associated with pMTG and angular gyrus). The WA group were impaired on both nonverbal and verbal comprehension assessments consistent with a generalised semantic impairment. This semantic deficit was most similar in nature to that of the semantic aphasia group suggestive of a disruption to semantic control processes. In addition, only the WA group showed a strong effect of input modality on comprehension, with accuracy decreasing considerably as acoustic-phonological requirements increased. These results deviate from traditional accounts which emphasise a single impairment and, instead, implicate two deficits underlying the comprehension disorder in WA.
\end{abstract}

(c) 2011 Elsevier Ltd. All rights reserved.

\section{Introduction}

Wernicke's contribution to the study of language and aphasia is undeniable. His identification of the neuroanatomical correlate of sensory (Wernicke's) aphasia and his interpretation of its role in comprehension continues to influence neurobiological models of language. Despite this, fundamental questions remain over the cause of the comprehension impairment in sensory aphasia (Wernicke's aphasia from here on, WA). This study examined three hypotheses about the comprehension impairment in WA by comparing their performance on a range of phonological and semantic assessments against two other comprehension-impaired clinical groups, semantic dementia (SD) and semantic aphasia (SA). SA refers to individuals with stroke aphasia who display a multi-modal semantic impairment (Head, 1926; Jefferies \& Lambon Ralph, 2006). SA and SD provide neuropsychological models of different types of comprehension impairments thereby providing a baseline against which the comprehension impairment in WA can

\footnotetext{
* Corresponding author at: Neuroscience and Aphasia Research Unit (NARU), Zochonis Building, School of Psychological Sciences, University of Manchester, Oxford Road, Manchester M13 9PL, UK. Tel.: +44 1612752551.

E-mail address: matt.lambon-ralph@manchester.ac.uk (M.A. Lambon Ralph).
}

be assessed. In the current study, we used a case-series comparison method to investigate the ways in which the comprehension impairment of WA is similar to that found in SA or in SD and also to reveal the features that are unique to WA.

WA is characterised by three core diagnostic criteria, impaired auditory comprehension, impaired repetition and fluent speech (Goodglass, Kaplan, \& Barresi, 2001a). Wernicke originally identified the neuroanatomical correlate of WA as a lesion to the posterior superior temporal gyrus (pSTG: Eggert, 1977). Outside these core behaviours, considerable heterogeneity in other language and cognitive behaviours has been noted which has been mirrored by variation in anatomical lesion correlates (e.g., Bogen \& Bogen, 1976). For example, variation in reading and writing skills and the extent and types of errors in spoken language is observed clinically and within reports of Wernicke's aphasia in the literature (Anzaki \& Izumi, 2001; Benson \& Ardila, 1996; Ellis, Miller, \& Sin, 1983; Lytton \& Brust, 1989; Warrington \& Leff, 2000). Although stroke aphasia is notoriously heterogeneous in nature, an additional source of this observed behavioural variation may be due to differences in the diagnostic methods, procedures and assessment tools used. Studies which define the inclusion criteria solely by the presence of a comprehension impairment and/or fluent speech run the risk of including a wider variety of clinical aphasia subtypes (including SD, transcortical sensory aphasia and 
conduction aphasia). Such variation in diagnosis and inclusion criteria has lead to difficulties in comparing results between studies and so this investigation used explicit diagnostic/inclusion criteria for WA in order to facilitate future generalisation.

Three hypotheses have been proposed to account for the comprehension impairment in WA: (1) a disruption to acoustic and/or phonological analysis; (2) a semantic impairment; or (3) a combined phonological-semantic impairment (referred to here as the dual hypothesis). The current study examined all three hypotheses in a case-series of relatively homogenous WA participants.

\subsection{The acoustic-phonological hypothesis}

Wernicke hypothesised that the posterior pSTG formed a store of auditory images for words (Wortklangbilder: Eggert, 1977). Damage to these auditory images would result in sounds being heard but the words would be meaningless (Eggert, 1977; Lichtheim, 1885). Later, this hypothesis was refined by Luria who proposed that an inability to perceive semantically relevant phonemic contrasts led to a deficit in phonemic decoding of the incoming acoustic stream (Luria, 1976; Luria \& Hutton, 1977). Thus, Luria's hypothesis implicates a more general deficit in acoustic analysis whereas Wernicke's interpretation implies impairment to the phonological representations themselves. Throughout this paper, this position will be referred to as the 'acoustic-phonological' hypothesis. This hypothesis has the additional attraction that it can account for both core impairments in WA: repetition as well as comprehension would be disrupted through a deficit in the acoustic-phonological analysis of spoken words.

Functional and structural neuroimaging studies have provided considerable evidence for the acoustic-phonological hypothesis. The left pSTG and surrounding areas, particularly the superior temporal sulcus (STS), are consistently activated in response to phonological stimuli and during tasks requiring phonological manipulation (e.g., Graves, Grabowski, Mehta, \& Gupta, 2008; Leff et al., 2009; Mummery, Ashburner, Scott, \& Wise, 1999; Narain et al., 2003; Saur, Kreher, Schnell, Kummerer, Kellmeyer, \& Vry, 2008; Wise et al., 2001). In line with Wernicke's original hypothesis, the pSTG in particular has been implicated in both production and comprehension networks (Buchsbaum, Hickok, \& Humphries, 2001; Heim \& Friederici, 2003), phonetically disambiguating the acoustic stream in comprehension (Glasser \& Rilling, 2008) and acting as an interface between auditory and motor representations for production/repetition (Warren, Wise, \& Warren, 2005).

Despite this, behavioural evidence for the acoustic-phonological hypothesis has been difficult to find in WA itself. In two experiments, Blumstein, Baker, \& Goodglass (1977) and Baker, Blumstein, \& Goodglass (1981) tested Luria's decoding deficit hypothesis. While individuals with WA displayed a deficit in auditory discrimination, as predicted, the degree of this impairment did not correlate significantly with the level of the comprehension deficit. Although this lack of a relationship between acoustic-phonological processing and comprehension performance was interpreted as counter to the acoustic-phonological hypothesis, it should be noted that, as stated by the authors, this null result might have reflected the small number of participants $(n=6)$. Indeed, a recent study utilising a new phonological discrimination task and a larger case-series of typical WA cases did find a relationship between acoustic-phonological processing and comprehension performance (Robson, Lambon Ralph, \& Sage, in press).

\subsection{The semantic hypothesis}

A second prominent hypothesis to account for the comprehension deficit in WA implicates disruption to a semantic level of analysis. Evidence for the semantic hypothesis has been gained from in-depth lesion studies as well as behavioural experiments. While the PSTG is considered by many to be the core of Wernicke's area, lesions associated with WA spread beyond this region in all directions but particularly into the angular gyrus (AG) and posterior middle temporal gyrus (pMTG) (Bogen \& Bogen, 1976). More recent evidence suggests that the pMTG is the region of greatest lesion overlap in chronic WA (Dronkers, Redfern, \& Ludy, 1995; Dronkers, Wilkins, Van Valin, Redfern, \& Jaeger, 2004; Ogar et al., 2011). Furthermore, lesions to the pSTG and surrounding areas, particularly the STS, may be more strongly associated with conduction aphasia (an aphasic disorder associated with impaired repetition but intact comprehension: Dronkers et al., 2004). Consistent with this view, a previous study found that in aphasic patients who present with nonverbal as well as verbal semantic impairments, the lesion extends from pSTG/STS to include PMTG (as well as inferior parietal areas: Chertkow, Bub, Deaudon, \& Whitehead, 1997). Functional neuroimaging strongly implicates the pMTG and AG in semantic processing, including conceptual and lexical retrieval (Binder, Desai, Graves, \& Conant, 2009; Cabeza \& Nyberg, 2000; Indefrey \& Levelt, 2004; Vigneau et al., 2006) and recent tractography studies have proposed the MTG to be the core node in the semantic network (Saur et al., 2008, 2010) as have TMS studies (Hoffman, Pobric, Drakesmith, \& Lambon Ralph, in press; Whitney, Kirk, O'Sullivan, Lambon Ralph, \& Jefferies, 2011). Under this hypothesis, a pSTG lesion would not be the root cause of the comprehension deficit in WA but would contribute to their impaired repetition as observed in conduction aphasia (Hickok, 2000).

Various behavioural studies also support the semantic hypothesis. Individuals with WA can present with concurrent nonverbal semantic impairments including difficulties with colour-to-picture matching (De Renzi, Faglioni, Scotti, \& Spinnler, 1972), picture-topicture matching (Cohen, Kelter, \& Woll, 1980) and drawing from memory (Gainotti, Silveri, Villa, \& Caltagirone, 1983). Recent explorations in other patient groups have shown that there are at least two ways in which semantic cognition may break down; semantic representations may be degraded as observed in SD or the regulatory processes governing the verbal and nonverbal use of semantic knowledge may be damaged, as found in SA (Bozeat, Lambon Ralph, Patterson, Garrard, \& Hodges, 2000; Jefferies \& Lambon Ralph, 2006; Rogers et al., 2004). There is now convergent evidence from fMRI, TMS and PET studies that the ventrolateral anterior temporal lobes underpin a modality-invariant representation to the formation of coherent concepts, such that this region acts as a core hub for the semantic "database" (Binney, Embleton, Jefferies, Parker, \& Lambon Ralph, 2010; Lambon Ralph, Sage, Jones, \& Mayberry, 2010; Patterson, Nestor, \& Rogers, 2007; Pobric, Jefferies, \& Lambon Ralph, 2010a; Visser, Jefferies, \& Ralph, 2010). In contrast, SA is associated with damage to prefrontal or temporoparietal regions, areas that have been implicated in semantic and cognitive control (Jefferies \& Lambon Ralph, 2006; Thompson-Schill, D’Esposito, Aguirre, \& Farah, 1997; Wagner, Pare-Blagoev, Clark, \& Poldrack, 2001). Given that the lesion sites implicated in WA are remote to the anterior temporal lobe and overlapping with temporoparietal areas then any general semantic impairment might be expected to pattern with the characteristics observed in posterior SA and less so with the features of SD (see below).

\subsection{The dual hypothesis}

A third hypothesis accounts for the comprehension impairment in WA in terms of a combined deficit in acoustic-phonological analysis and semantic processing (Baker et al., 1981), hereafter referred to as the dual hypothesis. There is some limited behavioural evidence for this theory. For example, in a four-choice spoken word-to-picture matching experiment with semantically related, phonologically related and unrelated distracters, WA patients were 
found to make both semantic and phonological errors (Baker et al., 1981). The dual hypothesis might also fit with the lesion location in WA and its varying extent. The lesion distribution in WA has been shown to include both superior and middle temporal regions (Bogen \& Bogen, 1976), which functional imaging studies have implicated in phonological and semantic processing, respectively. This lesion distribution raises the question of whether both acoustic-phonological and semantic analysis have to be disrupted (as in the dual hypothesis) in order to produce the comprehension behaviours observed in WA or whether it is possible to account for the comprehension impairment in WA through either disrupted acoustic-phonological or semantic processing alone as a result of smaller lesions isolated to superior or middle temporal regions.

\subsection{The case-series comparison approach}

This study used a case-series comparison design in order to investigate each of the three hypotheses. This method allows one to observe whether group patterns hold true across severity at an individual level as well as allowing for direct comparison between different clinical groups by assessing all cases with the same materials. This design has been employed to reveal insights into the nature of the semantic impairment across groups such as semantic dementia, semantic aphasia and herpes simplex encephalitis (Corbett, Jefferies, Ehsan, \& Ralph, 2009; Jefferies \& Lambon Ralph, 2006; Lambon Ralph, Lowe, \& Rogers, 2007). Comparisons of semantic dementia (SD) and semantic aphasia (post-stroke aphasia resulting in multimodal semantic impairments: SA) have been previously undertaken by Lambon Ralph and colleagues (Corbett et al., 2009; Jefferies \& Lambon Ralph, 2006). Jefferies and Lambon Ralph (2006) compared participants $\mathrm{SD}$, resulting from atrophy of the bilateral anterior temporal lobes and participants with SA resulting from inferior frontal and/or temporoparietal infarction. Despite equivalent overall accuracy the two groups demonstrated clear, qualitative differences in semantic performance, in line with the very different brain lesions and locations. For example, the SD group displayed strong sensitivity to item familiarity and high consistency between all types of semantic test. In contrast, the SA cases displayed no familiarity effect and consistency was found only between tasks with similar task demands. The SA group displayed a correlation between semantic tasks and executive assessments whereas no such correlation was found in the SD group. These and other results suggested that SD reflected degradation of the modality-invariant hub for semantic representations (Lambon Ralph et al., 2010; Patterson et al., 2007; Pobric, Jefferies, \& Lambon Ralph, 2010b) whereas SA reflected impaired executive processes which normally regulate the use of semantic information for task- and time-appropriate semantically driven behaviour (Corbett et al., 2009; Jefferies \& Lambon Ralph, 2006; Noonan, Jefferies, Corbett, \& Lambon Ralph, 2010) - which fits with Head's original description (1926). The present study compared a WA case-series to the SA and SD data reported previously by Jefferies and Lambon Ralph (2006). The SA and SD data provide neuropsychological models of expected performance across the various semantic tests in the face of two types of multimodal semantic impairment. If the WA participants demonstrate modality-differences (as predicted by the acoustic-phonological and dual hypotheses, see below) then the SA/SD groups provide an important contrastive dataset and allow us to be more certain that any performance differences are not due instead to task difficulty or participant severity. Furthermore, if a multimodal semantic impairment contributes at least in part to the comprehension deficit in WA, then these direct comparisons allow us to consider whether this reflects faulty control mechanisms or degraded semantic representations.

\subsection{Testing the hypotheses}

Each of the hypotheses makes the following predictions about how the WA group should perform on semantic tests in relation to the SA and SD groups.

(a) The acoustic-phonological hypothesis: predicts that the three groups (WA, SA and SD) should all exhibit deficits in semantic assessments which require acoustic-phonological input whereas the WA participants, unlike the two other groups, should not show impairment on nonverbal semantic assessments.

(b) The semantic hypothesis: predicts that there will be no contribution of acoustic-phonological disruption to comprehension and, therefore, all three groups should show overlapping performance on all semantic assessments independent of task input modality.

(c) The dual hypothesis: predicts that the WA group should show a nonverbal semantic impairment like the SA or SD groups with an additional, disproportionate impairment on assessments which rely on acoustic-phonological input.

Both the dual hypothesis and the semantic hypothesis implicate a semantic impairment. As noted above, previous studies have shown that the qualitative nature of semantic impairment can vary across patient groups (e.g., semantic dementia vs. semantic aphasia). Accordingly, we analysed the nature of the semantic impairment in the WA group. The predictive value of item familiarity, the correlation with executive skills and the degree of item consistency between assessments provide diagnostic features which indicate whether the semantic impairment in WA corresponds more closely to that observed in the SA or the SD.

\section{Method}

\subsection{WA participants and lesion analysis}

This research was carried out with full approval of the local ethics committee. Nine participants with chronic WA were recruited from speech and language therapy services in the North of England. Table 1 contains background screening assessment and biographical details. All tables present participants ordered by the degree of comprehension impairment as measured by the Boston Diagnostic Aphasia Examination - Short Form (BDAE) (Goodglass, Kaplan, \& Barresi, 2001b). The initial recruitment guidance sought participants with comprehension impairments, fluent speech and errors on repetition or naming. Following referral, participants were formally screened and diagnosed using the BDAE (Goodglass et al., 2001b). Patients were required to show a comprehension impairment below the 45th percentile and a sentence repetition impairment below the 65th percentile on the BDAE. However, in the current case series all participants fell into the bottom 20th percentile on repetition and comprehension subtests. In addition, average phrase length in everyday speech was required to be above six words. In structured picture description paraphasias had to occur at least every few utterances. These paraphasias could be semantic or phonological. In fact, all patients primarily produced phonological or neologistic paraphasias with limited semantic errors.

Neuroimaging data were acquired for all the WA participants. Four participants (LB, EL, DM, RD) consented to and were able to take part in T1w structural MRI scanning. Chronic MRI scans were acquired on a $3 \mathrm{~T}$ Philips Achieva scanner with an eight element SENSE head coil with a sense factor of 2.5. An inversion recovery sequence produced a $256 \times 256$ matrix of 128 transverse slices with $1 \mathrm{~mm}^{3}$ voxels. For the remaining participants CT scans taken at an acute stage as part of routine clinical practice were obtained. CT scans were analysed using templates for analysis of CT scans (Damasio \& Damasio, 1989). For those with MRI scans, lesions were detected using the automated lesion detection procedure described in Seghier, Ramlackhansingh, Crinion, Leff, \& Price (2008). Table 2 summarises relevant language processing regions and the extent to which these were affected over the case series.

Comparing the lesion distribution in current WA aphasia cases to the lesion distribution in the ten SA and ten SD cases previously reported in Jefferies and Lambon Ralph (2006), partially distinct and partially overlapping patterns of cortical involvement could be observed. The ten SA cases all showed left temporoparietal and/or prefrontal lesions on MRI and/or CT scans (see Table 2) and the 
Table 1

Demographic information and BDAE - Short Form results for the WA group.

\begin{tabular}{|c|c|c|c|c|c|c|c|}
\hline Case & Age & Sex & $\begin{array}{l}\text { Time post-onset at } \\
\text { testing }\end{array}$ & $\begin{array}{l}\text { BDAE } \\
\text { comprehension } \\
\text { (percentile) }\end{array}$ & $\begin{array}{l}\text { BDAE fluency } \\
\text { (percentile) }\end{array}$ & $\begin{array}{l}\text { BDAE sentence repetition } \\
\text { (percentile) }\end{array}$ & $\begin{array}{l}\text { BDAE word repetition } \\
\text { (percentile) }\end{array}$ \\
\hline $\mathrm{AB}$ & 85 & $\mathrm{~F}$ & 6-12 months & $<1$ & 75 & $<1$ & $<1$ \\
\hline LB & 78 & $\mathrm{~F}$ & 6 years & 5 & 68 & 5 & 15 \\
\hline EL & 60 & $\mathrm{M}$ & 4-6 months & 6.5 & 84 & 5 & 10 \\
\hline MR & 64 & M & 5-7 months & 10 & 68 & 15 & 15 \\
\hline RD & 86 & $\mathrm{M}$ & $12-14$ months & 10 & 80 & 5 & 10 \\
\hline DH & 74 & $\mathrm{M}$ & 5-7 months & 12 & 51 & 10 & 15 \\
\hline DM & 86 & M & 5-7 months & 13 & 57 & $<1$ & $<1$ \\
\hline$A C$ & 53 & $\mathrm{M}$ & 5-9 months & 15 & 68 & $<1$ & $<1$ \\
\hline HS & 81 & $\mathrm{~F}$ & 6-8 months & 18 & 75 & 15 & 15 \\
\hline
\end{tabular}

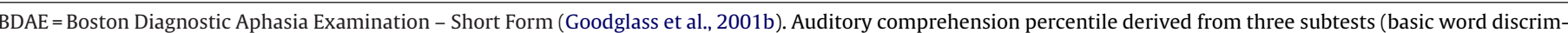

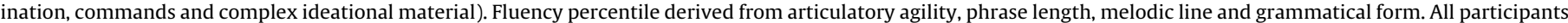
fell within the WA profile defined by the BDAE.

Table 2

Lesion summary for the WA group and SA group taken from (Noonan et al., 2010).

\begin{tabular}{|c|c|c|c|c|c|c|c|}
\hline Participant & Type of scan & pSTG & SMG & pMTG & sOT & AG & $\mathrm{TP}$ \\
\hline & & BA 22 & BA 40 & BA 21 & BA 37 & BA 39 & BA 38 \\
\hline$A B$ & Acute CT & + & - & + & + & - & - \\
\hline LB & Chronic MRI & + & + & + & + & + & - \\
\hline EL & Chronic MRI & + & + & + & - & + & - \\
\hline MR & Acute CT & + & - & + & + & - & - \\
\hline RD & Chronic MRI & + & + & + & - & + & - \\
\hline DH & Acute CT & + & + & + & + & - & - \\
\hline DM & Chronic MRI & + & + & + & + & + & - \\
\hline$A C$ & Acute CT & + & + & - & - & - & - \\
\hline HS & Acute CT & + & - & + & - & + & - \\
\hline \multicolumn{2}{|c|}{ \% WA cases with damage } & 100 & 66 & 89 & 55 & 55 & 0 \\
\hline \multicolumn{2}{|c|}{$\%$ SA cases with damage $(n=10)$} & 33 & 33 & 56 & 78 & 44 & 0 \\
\hline
\end{tabular}

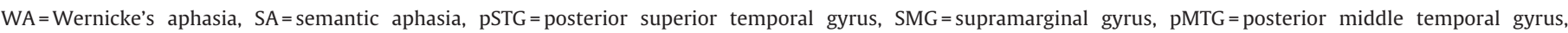
$\mathrm{sOT}=$ superior occipito-temporal area, $\mathrm{AG}=$ angular gyrus, $\mathrm{TP}=$ temporal pole, $\mathrm{NA}=$ not available. No neuroimaging data were available for one participant with WA (LB).

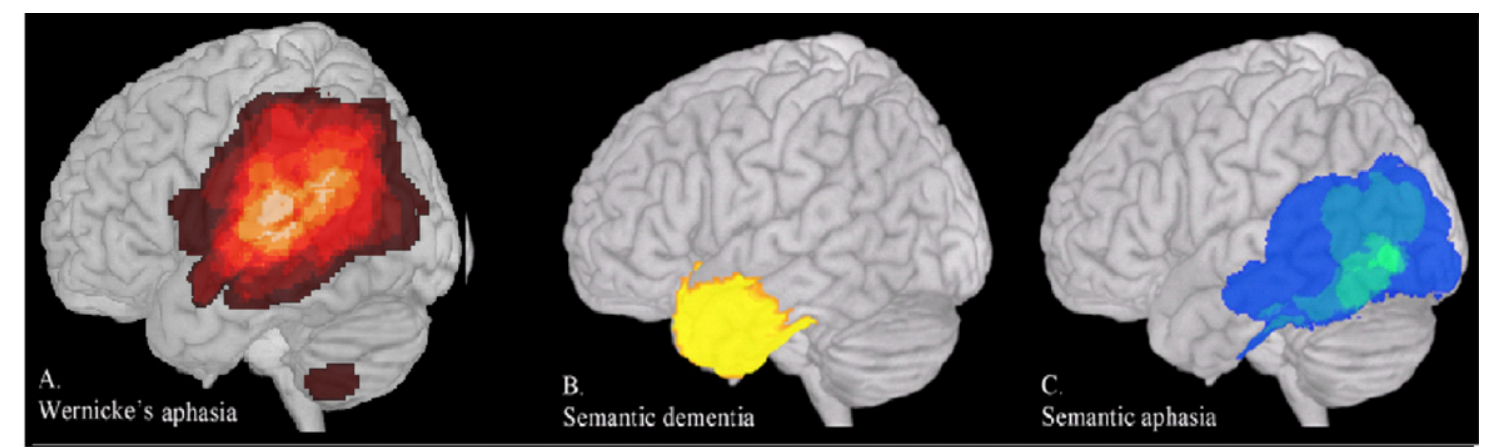

Footnote: Panel A shows the lesion overlap in the Wernicke's aphasia group, the lightest colours reflecting the areas of maximal lesion overlap. Panel B represents the hypometabolism in semantic dementia (reported by Nestor et al, 2006). Panel C shows the lesion overlap for the posterior subgroup of semantic aphasia (from Noonan et al., 2010), the lightest colours reflecting maximal lesion overlap.

Fig. 1. Wernicke's aphasia, semantic dementia and posterior semantic aphasia lesion/hypometabolism overlap maps.

ten cases of SD showed the typical bilateral atrophy centred on the inferolateral aspect of anterior temporal lobes (Jefferies \& Lambon Ralph, 2006). The WA cases showed some overlap with the SA cases with posterior temporo-parietal lesions, but more consistent and extensive involvement of superior temporal regions. Fig. 1 provides lesion/hypometabolism overlap maps for the three groups. ${ }^{1}$

1 The lesion overlap maps were produced using the MRIcro program (Rorden, 2005). Lesions analysed from CT scans for the WA cases and CT and MRI scans for the SA cases were manually drawn onto a standardised MNI template. For the WA lesion overlap map, manually drawn lesions from CT scans were combined with lesions identified from MRI scans using the automated lesion detection procedure (Seghier et al. (2008). The region highlighted for SD reflects the area of hypometabolism described by Nestor et al. (2006).

\subsection{Assessments}

Data were collected from the WA group to compare directly with existing results from the SA and SD cases. A number of additional assessments were performed in the WA group alone in order to investigate phonological processing and modality effects in greater detail.

\subsubsection{Phonological assessments}

The WA cases were tested on five assessments of phonological integrity. Word and nonword repetition were assessed using the 9th subtest from the Psycholinguistic Assessment of Language Processing in Aphasia (PALPA: Kay, Coltheart, \& Lesser, 1992). Forward digit span was used as a measure of phonological short term memory. Nonword and word minimal pair discrimination (PALPA 1 \& 2: Kay et al., 1992) was used to examine processing of phonological input without spoken output. Comparative word repetition data were available for the SA group and digit span data were available for all three groups. 


\subsubsection{Semantic memory assessments}

All semantic assessments were carried out in at least two modalities to allow for comparison of access to and manipulation of the same concepts across different input modalities. All assessments required a nonverbal response (pointing) only.

Pyramids and Palm Trees (PPT, Howard \& Patterson, 1992): is a standardised test of semantic association in which the participant decides which of two items is most semantically related to a probe item. This was performed in both the three picture and three written word formats.

Environmental sounds battery (ESB): Four tests from the battery (Bozeat et al. 2000) were administered: (a) environmental sound-to-picture matching; (b) environmental sound-to-written word matching; (c) spoken word-to-picture matching (sWPM); and (d) written word-to-picture matching (wWPM). In this 48-item test battery the participant matched the target stimulus (sound or word) to the correct picture which was placed within 9 semantically related items. wWPM data were not available for the SD group.

Cambridge semantic battery: This 64-item battery (Bozeat et al., 2000) assesses knowledge of the same concepts over different types of semantic judgments and modalities. Two versions (picture and written word) of the Camel and Cactus Test (CCT) were performed. This test required the participant to identify which of four semantically related items was the most related to a probe item. The CCT may be more sensitive to some types of semantic impairments than the PPT due to the greater number of choices per trial (Jefferies \& Lambon Ralph, 2006). Two additional subtests from the 64-item battery were administered; spoken word-to-picture matching (sWPM) and written word-to-picture matching (wWPM). ${ }^{2}$ Participants matched a spoken or written word to a picture from a group of ten semantically related items. Only sWPM data were available for the SA and SD groups.

\subsubsection{Familiarity ratings}

Previous studies provided concept familiarity ratings (Garrard, Lambon Ralph, Hodges, \& Patterson, 2001) for the 64-item battery and concept and sound familiarity ratings for the environmental sounds battery (Bozeat et al., 2000).

\subsubsection{Other cognitive skills}

Cognitive assessments used captured a range of nonverbal executive processes. The WA group were assessed on the Coloured Progressive Matrices (Raven, 1962), a commonly used test of nonverbal reasoning and spatial perception, and selected subtests from the Visual Object and Space Perception Battery (VOSP, Warrington \& James, 1991) - dot counting, position discrimination, number location and cube analysis, assessments of visual space perception and discrimination. Comparative data were available from both other groups for these assessments. The WA group were examined on three additional tests of attention and executive skills for which data were also available for the SA group - the Wisconsin Card Sort test (WCST, Grant \& Berg, 1993) to further assess nonverbal reasoning and inhibition, the Brixton Spatial Rule Attainment task (Burgess \& Shallice, 1997) which assesses monitoring and detection of sequence changes, and an auditory attention task, the Elevator Counting subtests, with and without distraction, from the Test of Everyday Attention (TEA, Robertson, Ward, Ridgeway, \& Nimmo-Smith, 1994).

\section{Results}

Mean group and individual data from the WA participants are presented alongside the mean and standard deviation data from the previously reported SA and SD cases for comparison. Phonological assessment results are presented in Table 3 and semantic memory assessment results in Table 4. Individual participant data for the SA and SD groups are available in Jefferies and Lambon Ralph (2006) and Bozeat et al. (2000), respectively.

\subsection{Phonological assessments}

The WA group were severely impaired on the four phonological measures: word repetition, nonword repetition, digit span and phonological discrimination (see Table 3). This result held for all members of the case-series across tasks requiring both phonological analysis and production (repetition and digit span) and input alone (phonological discrimination). Half of the group (AB, LB, EL and $\mathrm{HS}$ ) performed within chance level on phonological minimal pair discrimination and 7/9 WA participants were within chance level for nonword minimal pair discrimination $(A B, L B, M R, D H$,

\footnotetext{
2 Note: both batteries (the environmental sound battery and the Cambridge semantic battery) included separate measures of spoken and written word-picture matching.
}

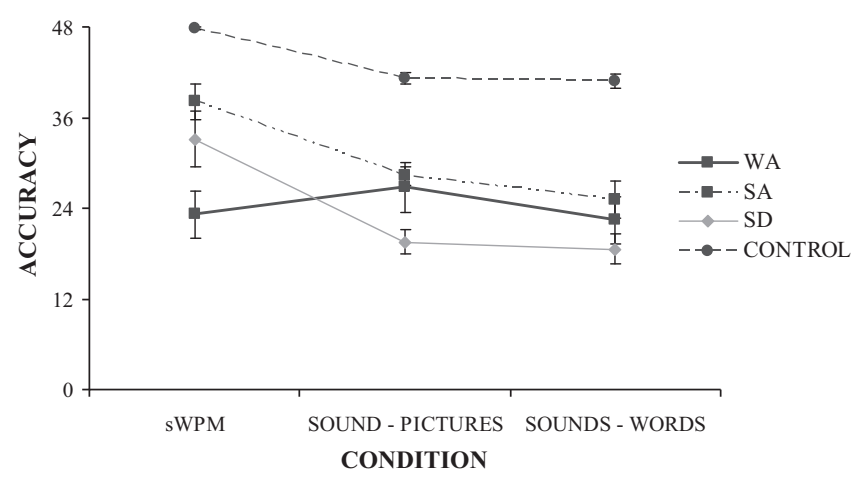

NB. $s W P M=$ spoken word picture matching, WA = Wernicke's aphasia, $\mathrm{SA}=$ semantic aphasia, $\mathrm{SD}=$ semantic dementia

Fig. 2. Environmental sounds battery results across patient groups.

DM, AC, HS). The WA group was clearly delineated from the SA and the SD groups on these phonological measures. The WA participants were significantly more impaired than the SA group on word repetition $\left(t_{(16)}=-5.2, p \leq 0.001\right)$ who scored within normal limits on this measure (group mean $=73.8$ ) when one SA participant with no verbal output was excluded (KA), this result should be treated with caution however as impaired single word repetition was an explicit inclusion criteria of the WA participants. There was an overall difference in performance on digit span between the three groups $(F(2,25)=27.5, p<0.001)$; the WA participants displayed significantly shorter digit spans than both the $S A\left(t_{(1)}=3.6\right.$, $p=0.002)$ and the $\mathrm{SD}\left(t_{(17)}=3.0, p=0.008\right)$ groups.

\subsection{Visual nonverbal semantic impairment}

As a group, the WA participants were impaired on both nonverbal semantic association assessments (picture PPT and picture CCT; see Table 4). A repeated measures ANOVA indicated that the degree of nonverbal semantic impairment was comparable across all three groups with no significant group effects (PPT: $F(2,26)<1, \mathrm{CCT}$ : $F(2,26)<1)$. At the group level, therefore, the acoustic-phonological hypothesis was insufficient to account for the comprehension impairment in WA because their performance was abnormal on both verbal and nonverbal versions of this semantic test. In addition, there was considerable variation within the WA case-series; two of the nine participants (RD and $A C$ ) performed within normal limits on the picture PPT and CCT, which might be indicative of differential semantic performance across modalities - a possibility investigated below.

\subsection{Modality effects - three group comparison}

A repeated measures ANOVA was used to investigate whether input modality affected accuracy in any of the groups. The groups were compared on the environmental sounds battery (ESB: spoken word-picture matching, sound-picture and sound-word conditions - see Fig. 2) and Cambridge semantic battery (CBB: spoken word-picture matching, picture CCT and word CCT conditions see Fig. 3 ). Significant condition $\times$ group interactions were found in both batteries (ESB: $F(4,52)=10.4, p<0.001$, CSB: $F(4,48)=5.0$, $p=0.002)$. These interactions were not found when the WA group was removed from the analyses (ESB: $F(2,34)=0.7, p=0.5$; CSB: $F(2,32)=1.8, p=0.2)$. Subsequent $t$-tests showed that the interaction in both batteries was driven by the WA group's poor performance in the spoken word-picture matching tests. This was significantly worse than the SA group (ESB: $t_{(17)}=3.9, p=0.001$; CSB: $\left.t_{(17)}=2.9, p=0.009\right)$ and the SD (ESB: $t_{(17)}=2.0, p=0.03$, 
Table 3

Phonological assessment results for the WA, SA and SD groups.

\begin{tabular}{|c|c|c|c|c|c|c|c|c|c|c|c|c|c|c|}
\hline Assessment & Max. & Normal cut-off & $\mathrm{AB}$ & LB & $\mathrm{EL}$ & MR & $\mathrm{RD}$ & $\mathrm{DH}$ & $\mathrm{DM}$ & $\mathrm{AC}$ & HS & Mean & SA mean (sd) & SD mean (sd) \\
\hline Word repetition $^{\mathrm{a}}$ & 80 & 73 & 20 & 7 & 14 & 6 & 7 & 36 & 0 & 6 & 42 & 15.3 & $65.6(22)$ & $\mathrm{NT}^{*}$ \\
\hline Nonword repetition $^{\mathrm{a}}$ & 80 & 62.5 & 11 & 0 & 0 & 3 & 0 & 30 & 0 & 4 & 12 & 6.67 & $\mathrm{NT}^{*}$ & $\mathrm{NT}^{*}$ \\
\hline Forward digit span & & & 3 & 2 & 2 & 2 & 1 & 2 & 0 & 2 & 2 & 1.78 & $4.4(1.7)$ & $6.5(1.4)$ \\
\hline Minimal pair discrimination ${ }^{\mathrm{a}}$ & 72 & 64 & 38 & 37 & 36 & 57 & 50 & 47 & 50 & 43 & 32 & 43.3 & $\mathrm{NT}^{*}$ & $\mathrm{NT}^{*}$ \\
\hline Nonword minimal pair discrimination ${ }^{\mathrm{a}}$ & 72 & 65 & 29 & 39 & 45 & 33 & 44 & 42 & 42 & 40 & 41 & 39.4 & $\mathrm{NT}^{*}$ & $\mathrm{NT}^{*}$ \\
\hline
\end{tabular}

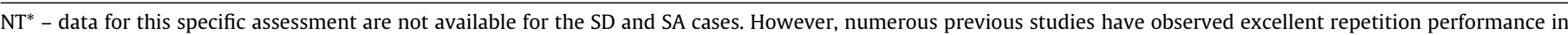
these two groups (Jefferies \& Lambon Ralph, 2006). Italicsed font indicates score within chance level, binomial test.

a Subtest 9 of the PALPA was used to measure word and nonword repetition (Psycholinguistic Assessment of Language Processing in Aphasia: Kay et al., 1992). Subtest

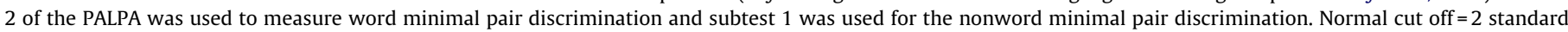
deviations below mean.

Table 4

Semantic memory assessment results for the WA, SA and SD groups.

\begin{tabular}{|c|c|c|c|c|c|c|c|c|c|c|c|c|c|c|}
\hline Assessment & Max. & Normal cut-off & $\mathrm{AB}$ & LB & EL & MR & $\mathrm{RD}$ & $\mathrm{DH}$ & $\mathrm{DM}$ & $\mathrm{AC}$ & HS & WA mean & SA mean (sd) & $\mathrm{SD}$ mean $(\mathrm{sd})$ \\
\hline PPT pictures & 52 & 49 & 34 & 48 & 48 & 50 & 50 & 39 & 43 & 49 & 40 & 44.6 & $40.1(6.7)$ & $40.5(10)$ \\
\hline PPT words & 52 & 49 & 28 & 42 & 36 & 39 & 52 & 28 & 40 & 46 & 35 & 38.4 & $41(4.9)$ & $38.7(10)$ \\
\hline CCT words & 64 & 57 & 18 & 43 & 36 & 46 & 55 & 22 & 40 & 48 & 23 & 36.8 & $37.1(10.1)$ & $37.1(16.1)$ \\
\hline Spoken word-picture & 64 & 64 & 23 & 26 & 30 & 32 & 47 & 41 & 45 & 47 & 34 & 36.1 & $49.7(10.8)$ & $45.4(19.4)$ \\
\hline Written word-picture & 64 & NA & 30 & 58 & 59 & 58 & 64 & 48 & 62 & 63 & 38 & 53.3 & NT & NT \\
\hline \multicolumn{15}{|c|}{ Environmental sounds battery } \\
\hline Sounds-picture & 48 & 36 & 10 & 25 & 30 & 20 & 39 & 30 & 35 & 36 & 16 & 26.8 & $28.3(3.7)$ & $19.5(6.2)$ \\
\hline Sounds-word & 48 & 34 & 9 & 26 & 24 & 17 & 32 & 18 & 22 & 40 & 14 & 22.4 & $25.1(7.5$ & $18.6(5.0)$ \\
\hline Spoken word-picture & 48 & 46.6 & 8 & 15 & 21 & 26 & 36 & 29 & 31 & 29 & 14 & 23.2 & $38.1(7.3)$ & $33.1(11.8)$ \\
\hline Written word-picture & 48 & NA & 25 & 41 & 45 & 40 & 46 & 43 & 40 & 42 & 28 & 38.9 & $39.1(8.0)$ & NT \\
\hline
\end{tabular}

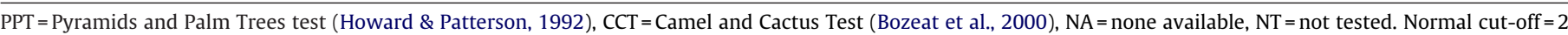
standard deviations below mean.

one-tailed). These results indicated that the WA group were uniquely affected by input modality, with especially poor auditoryverbal performance, providing support for the dual hypothesis.

\subsection{Modality effects in Wernicke's aphasia}

Modality effects in the WA group were investigated in depth. The results indicated that impaired acoustic-phonological analysis made a significant contribution to the comprehension impairment in WA.

\subsubsection{Written vs. pictorial}

Performance on the word and picture versions of PPT and CCT were compared. The WA group were significantly worse on the written than picture versions of the PPT $\left(t_{(8)}=4.0, p=0.004\right.$, two-tailed) and the CCT $\left(t_{(8)}=1.9, p=0.04\right.$, one-tailed). All bar one participant (RD) scored lower on the written than the picture version of the PPT and this difference was significant at the

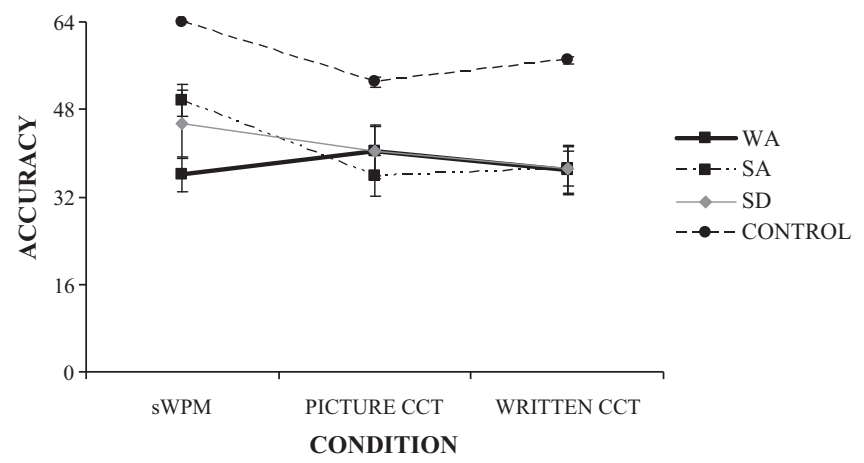

NB. $\mathrm{sWPM}=$ spoken word picture matching, $\mathrm{CCT}=\mathrm{Camel}$ and Cactus Test, WA = Wernicke's aphasia, $\mathrm{SA}=$ semantic aphasia, $\mathrm{SD}=$ semantic dementia

Fig. 3. Cambridge semantic battery results across patient groups. individual level for three participants (two-tailed McNemar tests, EL: $p=0.004$, MR: $p=0.001$, DH: $p=0.03$ ). Two cases (EL and AC) exhibited a significant difference between the written and the picture CCT (two-tailed McNemar tests, EL: $p=0.01$, AC: $p=0.04$ ).

\subsubsection{Spoken vs. written verbal}

Spoken word-picture matching (sWPM) and written word-picture matching (wWPM) from the Cambridge semantic battery and environmental sounds battery were compared. The WA group performed significantly worse on the sWPM than wWPM in both the environmental sounds battery $\left(t_{(8)}=8.1, p<0.001\right.$, twotailed) and the Cambridge semantic battery $\left(t_{(8)}=5.1, p=0.01\right)$. McNemar two-tailed tests showed that this difference was significant at the individual level for eight of the nine participants in the environmental sounds battery and for six participants in the Cambridge semantic battery (ESB; AB $p<0.001$, LB $p<0.001$, EL $p<0.001$, MR $p=0.003$, RD $p=0.002$, DH $p=0.003$, DM $p=0.035$, AC $p=0.004$. CSB; LB $p<0.0001$, EL $p<0.0001$, MR $p<0.0001$, RD $p<0.0001$, DM $p=0.0001, \mathrm{AC} p<0.0001)$. Crucially the two participants (RD and AC) who scored within normal limits on the picture CCT were both impaired on the sWPM from the Cambridge semantic battery. This is consistent with modality-specific differences as per the acoustic-phonological and dual hypotheses.

\subsubsection{Speech vs. environmental sounds}

Modality differences were investigated within the auditory domain by comparing sWPM to environmental sound-picture matching. The WA group were impaired in both conditions. The group was significantly more impaired in the sWPM condition than the sound-picture matching condition $\left(t_{(8)}=2.2, p=0.029\right.$, onetailed). This result becomes more noteworthy when the pattern of performance in the control population is taken into account. Elderly control participants $(n=20)$ were assessed on the environmental sounds battery for previous studies (Bozeat et al., 2000; Jefferies \& Lambon Ralph, 2006). The control participants performed significantly worse on the sound-to-picture matching task than the $\operatorname{sWPM} \operatorname{task}\left(t_{(38)}=11.3, p<0.0001\right)$. Thus the WA participants were 
Table 5

Executive assessment results for the WA, SA and SD groups.

\begin{tabular}{|c|c|c|c|c|c|c|c|c|c|c|c|c|c|c|}
\hline \multirow[t]{2}{*}{ Assessment } & \multirow[t]{2}{*}{ Max. } & \multirow{2}{*}{$\begin{array}{l}\text { Normal } \\
\text { cut-off }\end{array}$} & \multicolumn{9}{|l|}{ WA } & \multirow{2}{*}{$\begin{array}{l}\text { WA } \\
\text { mean }\end{array}$} & \multirow[t]{2}{*}{ SA mean (sd) } & \multirow[t]{2}{*}{ SD mean $(\mathrm{sd})$} \\
\hline & & & $\mathrm{AB}$ & LB & EL & MR & $\mathrm{RD}$ & $\mathrm{DH}$ & $\mathrm{DM}$ & AC & HS & & & \\
\hline Raven's coloured matrices (centile) & & & 50 & 50 & 75 & 90 & 75 & 50 & 50 & 75 & 10 & & & \\
\hline VOSP dot counting & 10 & 8 & 6 & 10 & 7 & 9 & 7 & 10 & 2 & 10 & NT & 7.6 & $8.2(2.5)$ & $10.0(0)$ \\
\hline VOSP position discrimination & 20 & 18 & 11 & 20 & 20 & 19 & 18 & 18 & 16 & 20 & NT & 17.8 & $17.6(2.1)$ & $19.5(1.2)$ \\
\hline VOSP number location & 10 & 7 & 1 & 7 & 10 & 5 & 9 & 8 & 7 & 9 & NT & 7.0 & $7.4(2.5)$ & $9.8(0.5)$ \\
\hline VOSP cube analysis & 20 & 6 & 6 & 6 & 6 & 6 & 3 & 5 & 1 & 9 & NT & 5.3 & $5.3(2.9)$ & $9.2(1.6)$ \\
\hline WCST (no. of categories) & 6 & 3 & 1 & 3 & 4 & 2 & 3 & 1 & 2 & 3 & 1 & 2.22 & $1.2(2.2)$ & NT \\
\hline Brixton spatial anticipation & 54 & 28 & 27 & 23 & 25 & 16 & 24 & 28 & 26 & 43 & 2 & 23.8 & $18(11)$ & NT \\
\hline TEA: without distraction & 7 & 6 & NT & 7 & 0 & 7 & 4 & 7 & 6 & 7 & 6 & 5.71 & $4.5(1.9)$ & NT \\
\hline TEA: with distraction & 10 & 3 & NT & 4 & 0 & 2 & 5 & 0 & 3 & 2 & 2 & 1.86 & $2.13(3.5)$ & NT \\
\hline
\end{tabular}

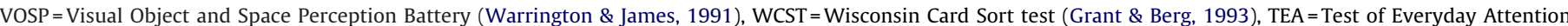
(Robertson et al., 1994).

more impaired on sWPM despite this being an easier task for non-neurologically impaired controls. Furthermore, the two participants with WA who scored within normal limits on nonverbal semantic assessments ( $R D$ and $A C$ ) were unimpaired on sound-topicture matching but were impaired on sWPM of the same items.

\subsection{The qualitative nature of the semantic impairment: is WA like $S A$ or $S D$ ?}

\subsubsection{Familiarity and item consistency}

In order to explore whether familiarity influenced the WA performance, logistic regression was used to predict accuracy on the subtests from the Cambridge semantic battery and the environmental sounds battery. The following factors were entered into the regressions: familiarity ratings from Garrard et al. (2001) and Bozeat et al. (2000), imageability ratings derived from the Neighbourhood Watch Programme (Davis, 2005) and participant. Item familiarity was not predictive of performance on any sub-test within the environmental sounds battery or Cambridge semantic battery (ESB: $s W P M$ Wald $=2.5, p=0.1$, wWPM Wald $=0.02$, $p=0.9$, sounds-pic Wald $=1, p=0.3$, sounds-words Wald $=1.9$, $p=0.2$; CSB: sWPM Wald $<0.0001, p=1$, wWPM Wald $<0.0001$, $p=1$, CCTpic Wald $<0.0001, p=1$, CCTword Wald $<0.0001, p=0.99$ ). Logistic regression was also used to assess item consistency across tasks (as per Jefferies \& Lambon Ralph, 2006). This was high where the semantic demands remained constant across tasks; the word-to-picture matching tasks predicted each other in both the environmental sounds (Wald=3.7, $p=0.05$ ) and the Cambridge semantic battery (Wald $=5.6, p=0.02$ ) and, similarly, the two versions of the CCT showed high item consistency ( Wald $=7.9$, $p=0.005)$. In all cases except one, item consistency was low between tasks where the semantic demands differed (e.g., CSB: sWPM and written CCT Wald $=0.4, p=0.5$ ). The one exception was that performance on wWPM significantly predicted performance on picture CCT (Wald $=9.7, p=0.002$ ). These qualitative patterns - no predictive value of familiarity, and task requirement related item consistency, resembled the patterns reported by Jefferies and Lambon Ralph (2006) for the SA group.

\subsubsection{Correlations with executive-cognitive skills}

Results of the executive and cognitive assessments for all three groups and individual results for WA participants are presented in Table 5. The WA group scores were within one point of normal limits on all four subtests of the VOSP (Warrington \& James, 1991). All WA participants fell within normal limits on the Coloured Progressive Matrices (Raven, 1962). Only four participants achieved three or more categories on the WCST (Grant \& Berg, 1993). All but one participant $(A C)$ were impaired on the Brixton Spatial Rule Attainment task (Burgess \& Shallice, 1997). On the auditory attention subtests of the TEA (Robertson et al., 1994), the WA group were just outside normal limits on counting without distraction but fell below normal limits when distraction was introduced.

Following Jefferies and Lambon Ralph (2006), factor analysis was used to derive separate executive and semantic summary scores for each WA participant in order to test the relationship between these factors (the analysis provides the best weightedaverage of the individual test scores in order to maximise the variance explained within attentional-executive or semantic scores). The executive component was derived from the WCST, Brixton Spatial Rule Attainment task and the Coloured Progressive Matrices and the semantic component from the picture PPT and the picture CCT. Other semantic assessments were not included in the semantic factor because of the potential confounding influence of impaired acoustic-phonological analysis on the results. In the WA group ( $n=9$ ), a significant correlation was found between the semantic and executive component (Pearson's correlation, $r=0.66$, $p=0.05$ ). A similar correlation between semantic and executive components were also found in the SA group $(n=10)$ reported by Jefferies and Lambon Ralph (2006).

\section{Discussion}

This study investigated the basis of the comprehension impairment in Wernicke's aphasia. Three hypotheses were tested via a detailed case-series analysis of Wernicke's aphasia (WA) and direct comparison to semantic dementia (SD) and semantic aphasia (SA). The three hypotheses were: (1) an acoustic-phonological analysis impairment alone; (2) an isolated semantic impairment; or (3) dual acoustic-phonological and semantic breakdown. The direct comparison with SA and SD allowed greater inferences to be drawn about the nature of the comprehension impairment in WA. The results clearly suggested that the comprehension impairment in WA was best accounted for by a dual breakdown in both acousticphonological analysis and semantic cognition.

The WA group were significantly more impaired on phonological tasks than both the SA and SD group and worse than control participants. An acoustic-phonological only deficit (Hypothesis 1) is insufficient to explain their comprehension impairment at a group level, however, because the majority of cases ( 7 participants) also displayed a nonverbal semantic impairment. This result concords with previous findings of nonverbal impairments in WA (e.g., Cohen et al., 1980; De Renzi et al., 1972; Gainotti \& Lemmo, 1976; Gainotti et al., 1983). Although a considerable nonverbal semantic impairment was observed at the group level, two WA participants were unimpaired on the nonverbal semantic assessments. These individuals were impaired on semantic assessments which required acoustic and/or phonological analysis such as spoken word-to-picture matching or written semantic association tests. These results suggested that there was a subset of individuals with 
Table 6

Summary of overlapping and distinguishing clinical features in WA, SA and SD.

\begin{tabular}{|c|c|c|c|}
\hline \multirow[t]{2}{*}{ Clinical feature } & \multicolumn{3}{|c|}{ Clinical group } \\
\hline & WA & SA & SD \\
\hline \multicolumn{4}{|l|}{ Lesion location } \\
\hline Anterior temporal lobes (bilaterally) & $\mathrm{x}$ & $\mathrm{x}$ & $\sqrt{ }$ \\
\hline Consistent lesion to left pSTG & $\sqrt{ }$ & $\mathrm{x}$ & $\mathrm{x}$ \\
\hline Lesion to pMTG/AG & $\sim$ & $\sim$ & $\mathrm{x}$ \\
\hline \multicolumn{4}{|l|}{ Key characteristics of comprehension impairment } \\
\hline Multimodal semantic impairment & $\sim$ & $\sqrt{ }$ & $\sqrt{ }$ \\
\hline Acoustic-phonological deficit & $\sqrt{ }$ & $\mathrm{x}$ & $\mathrm{x}$ \\
\hline \multicolumn{4}{|l|}{ Patterns within semantic performance } \\
\hline Item familiarity & $\mathrm{x}$ & $\mathrm{x}$ & $\sqrt{ }$ \\
\hline Item consistency & $\sim$ & $\sim$ & $\sqrt{ }$ \\
\hline Correlation with attentional-executive assessments & $\sqrt{ }$ & $\sqrt{ }$ & $\mathrm{x}$ \\
\hline
\end{tabular}

WA = Wernicke's aphasia, $\mathrm{SA}=$ semantic aphasia, $\mathrm{SD}=$ semantic dementia, $\mathrm{X}=$ feature not present, $\sqrt{ }=$ feature present, $\sim=$ features present in specific circumstances,

WA whose comprehension impairment was due solely to disrupted acoustic-phonological analysis.

Both the semantic and the dual hypotheses predicted a nonverbal semantic impairment. To distinguish between the two hypotheses, the variation of performance across input modalities was assessed in the WA group and compared to the SA and SD participants. Strong modality differences were observed in the WA group: (a) verbal worse than nonverbal semantic performance; and (b) particularly poor performance on semantic tasks requiring acoustic-phonological analysis. In summary, the comprehension impairment in WA is, in the majority of cases, best accounted for by the dual hypothesis. In comparison to the SD and SA groups, the acoustic-phonological impairment made a unique and significant contribution to the comprehension impairment in WA. Qualitative analysis found that the WA group showed a semantic impairment similar in nature to that of the SA group; proposed to reflect deregulated semantic control (which refers to executive control mechanisms - e.g., inhibition, retrieval, selection - that interact with semantic representations in order to generate task- and timeappropriate behaviour: Corbett et al., 2009; Jefferies \& Lambon Ralph, 2006); see also Head (1926). Table 6 provides a summary of the overlapping and distinguishing features between WA, SA and SD.

Fig. 4 provides a visual summary of how the WA and SA groups fit into the dual-dimension hypothesis-defined on the basis

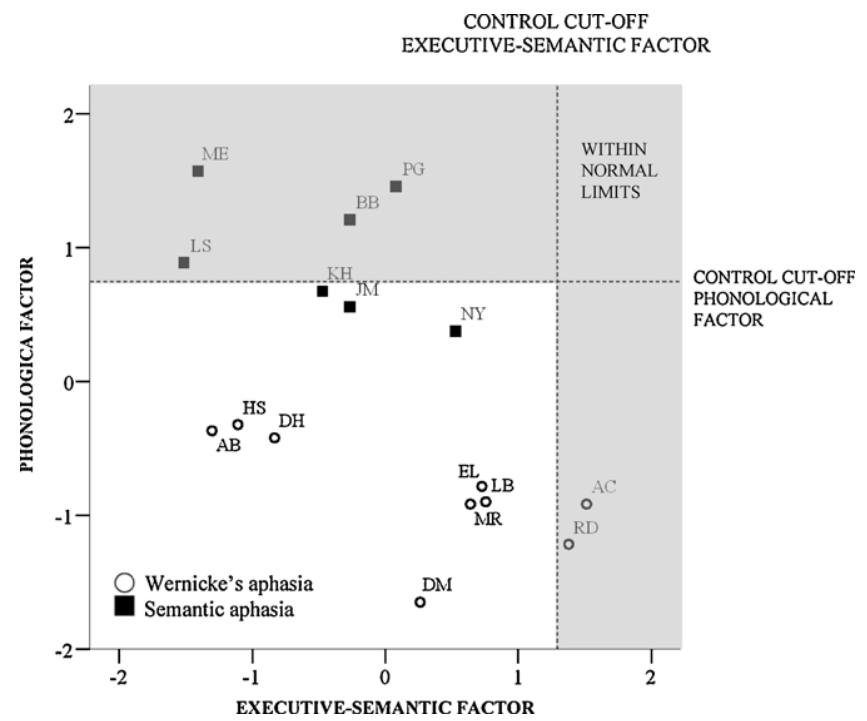

Fig. 4. Scatter plot to show the WA and SA participants' abilities along executivesemantic and acoustic-phonological dimensions. of acoustic-phonological abilities and semantic control. ${ }^{3}$ While there is important inter-individual variation in the SA group on semantic-executive measures, they fall within or at least very close to the normal range on the acoustic-phonological dimension. The same semantic-executive variation is demonstrated amongst the WA cases but, in addition, they occupy a very different (impaired) part of the acoustic-phonological dimension. This acoustic-phonological score was derived from repetition and digit span measures for which overlapping data was available between the WA and SA groups. Impaired repetition is part of the diagnostic criteria of Wernicke's aphasia and therefore low scores on this measure are expected. However, these results converge with highly impaired performance on phonological discrimination and significant modality differences between spoken and written wordto-picture matching, both assessing analysis of input phonology. That individuals with WA performed mostly at chance level on the minimal pair tests of phonological discrimination is problematic for analysis. This poor performance has been noted in previous work with WA (Morris, Franklin, Ellis, Turner, \& Bailey, 1996) and is likely to reflect both the method of presentation and stimuli consisting of inaccessible contrasts (Robson et al., in press). Overall, therefore, an acoustic-phonological impairment is the necessary impairment underlying the comprehension deficit in WA, and in some cases an isolated acoustic-phonological impairment is sufficient to cause WA. However, in the majority of cases there is an additive role of impaired semantic processing. These combined deficits lead to the severe comprehension difficulties seen in this group (all participants were in the bottom 20th percentile on BDAE comprehension measures) and the difficulties experienced in clinical remediation attempts.

\subsection{Comparison to lesions}

The behavioural results from this study correspond to the lesion distributions in the three clinical groups. The lesion distribution in the WA patients and posterior SA subgroup overlapped in areas associated with semantic processing, but in no case did lesions extend to the anterior temporal lobes where the focus of atrophy and hypometabolism is found in SD (Table 2 and Fig. 1). The WA and posterior SA cases both showed a considerable degree of involvement of posterior middle temporal regions and the angular gyrus, however, these two groups different in that the WA group showed consistent involvement of the posterior superior temporal gyrus and the SA group showed a greater degree of involvement of the superior posterior occipito-temporal region. The middle temporal region and angular gyrus have been identified repeatedly in neuroimaging studies as components of a distributed semantic network (Binder et al., 2009; Cabeza \& Nyberg, 2000; Vigneau et al., 2006; Visser et al., 2010) and the correspondence of lesions underpinning WA and SA groups fits with the qualitatively similar semantic impairment observed in both groups. Similarly, only the WA group exhibited an effect of impaired phonological analysis on comprehension, reflective of the greater degree of superior temporal lobe involvement. This pattern replicates a previous comparative study of aphasic patients with comprehension impairments. Those with comprehension impairment limited to the verbal domain had smaller pSTG-centred lesions whereas patients

\footnotetext{
${ }^{3}$ Fig. 4 was created using a principal component analysis to derive a combined measure of executive and semantic skills (from Ravens Coloured Progressive Matrices, Wisconsin Card Sorting Test, Brixton Spatial Rule Attainment task, Camel and Cactus Tests and Pyramids and Palm Trees Tests) and a separate measure of phonological ability (from word repetition from the Psycholinguistic Assessment of Language Processing in Aphasia and auditory digit span). The control cut-off for all the assessments was added into the analysis to derive the cut-off scores.
} 
with multimodal comprehension impairment had larger lesions that extended into pMTG and inferior parietal areas (Chertkow et al., 1997). Although the lesion-behaviour pattern in this study converges with previous neuropsychological evidence and current functional imaging evidence, some degree of caution should be taken. Only half of the WA group had chronic scanning data available and, therefore, the extent of the overlap between the WA group and the other clinical groups may be underestimated as the acute CT scans may not have captured the full extent of the lesion.

\subsection{Clarifying the dual hypothesis}

The dual hypothesis is currently underspecified. This study has been able to provide evidence to expound which elements of semantic cognition are disrupted, by showing that the executivesemantic impairment in WA was similar in nature to that in SA. Inferences into the exact nature of the acoustic-phonological disruption are more difficult to draw from the current data. However, some insights can be gleaned which further research could explore in more detail.

One question that can be addressed is whether the data from this study concord with the somewhat different approaches to the acoustic-phonological deficit proposed by Wernicke and Luria. Wernicke's theory emphasised a core linguistic deficit of phonological word forms (Eggert, 1977) whereas Luria's account emphasised a generalised deficit in psychoacoustic analysis which leads to impaired phonological disambiguation from the acoustic stream. The results from this study were more consistent with Wernicke's original interpretation. The WA group showed greater impairment in written semantic association tasks than in pictorial ones, a pattern which would not be expected if their impairment was purely acoustic/perceptual in nature, such as that seen in individuals with pure word deafness who are unimpaired at accessing semantic representations from written input (Buchman, Garron, Trost-Cardamone, Wichter, \& Schwartz, 1986). The group was significantly more impaired on spoken word-picture matching than sound-picture matching of the same items even though soundto-picture matching is a more difficult task. Further to this, the two participants (AC and RD) who were unimpaired on visual nonverbal semantics also performed within normal limits on sound-to-picture matching but outside the normal limits on spoken word-to-picture matching indicating their acoustic analysis of nonverbal sounds was much better or even intact. These results must be interpreted with caution, however, as the spoken words and environmental sounds are not matched for acoustic complexity and presentation time. The nature of the acoustic-phonological impairment requires further investigation using controlled nonverbal auditory stimuli.

\subsection{Dual hypothesis explains behavioural heterogeneity}

The notion that more than one deficit is required to account for the behavioural profile of WA has been raised before. Hickok (2000) suggested that the comprehension impairment in WA could be accounted for by an impairment to post-perceptual semantic processes, while impaired repetition would be accounted for by disrupted acoustic-motor integration. Under this hypothesis, there is a one-to-one relationship between symptom and cause. The approach taken by the current study differs somewhat in that a single impairment (in this case comprehension) results from dual underlying causes. This approach has the ability to account for the modality differences found in the WA group and the modalityequivalent effects in SA and SD, which are more difficult to explain through a one symptom-one cause approach. These dual deficits will also combine to impair speech output and repetition as well as comprehension. Thus both receptive and expressive symptoms arise from the same underlying dual mechanism. The dual hypothesis also has the capacity to explain much of the behavioural heterogeneity found in WA (see Fig. 4). The severity of individual WA patient's comprehension impairment reflects the combination of impaired acoustic-phonology (present across all WA cases) plus the varying degree of additional semantic processing deficits.

\section{Acknowledgements}

We would like to thank the participants and their relatives/carers for their assistance in this study, and the speech and language therapists in the North of England who have referred participants to this project. We would like to thank Paul Hoffman for his contribution to the creation of the lesion maps as well as Roland Zahn, James Keidel and Richard Binney for suggestions and discussions. This work was supported by a Stroke Association Allied Health Professional Research Bursary (TSAB2008/01) and MRC Programme Grants (G0501632 \& MR/J004146/1).

\section{References}

Anzaki, F., \& Izumi, S. (2001). Differences between conduction aphasia and Wernicke's aphasia. Tokai Journal of Experimental \& Clinical Medicine, 26(2), 45-61.

Baker, E., Blumstein, S. E., \& Goodglass, H. (1981). Interaction between phonological and semantic factors in auditory comprehension. Neuropsychologia, 19, 1-15.

Benson, F. D., \& Ardila, A. (1996). Aphasia: A clinical perspective. New York: Oxford University Press.

Binder, J. R., Desai, R. H., Graves, W. W., \& Conant, L. L. (2009). Where is the semantic system? A critical review and meta-analysis of 120 functional neuroimaging studies. Cerebral Cortex, 19(12), 2767-2796.

Binney, R. J., Embleton, K. V., Jefferies, E., Parker, G. J., \& Lambon Ralph, M. A. (2010) The inferolateral aspects of the anterior temporal lobe are crucial in semantic memory: Evidence from a novel direct comparison of distortion-corrected fMRI, rTMS and semantic dementia. Cerebral Cortex, 20, 2728-2738.

Blumstein, S. E., Baker, E., \& Goodglass, H. (1977). Phonological factors in auditory comprehension in aphasia. Neuropsychologia, 15, 19-30.

Bogen, J. E., \& Bogen, G. M. (1976). Wernicke's region - Where is it? Annals of the New York Academy of Sciences, 280, 834-843.

Bozeat, S., Lambon Ralph, M. A., Patterson, K., Garrard, P., \& Hodges, J. R. (2000) Non-verbal semantic impairment in semantic dementia. Neuropsychologia, 38 1207-1215.

Buchman, A. S., Garron, D. C., Trost-Cardamone, J. E., Wichter, M. D., \& Schwartz, M. (1986). Word deafness: One hundred years later. Journal of Neurology, Neurosurgery and Psychiatry, 49, 489-499.

Buchsbaum, B. R., Hickok, G., \& Humphries, C. (2001). Role of left posterior superior temporal gyrus in phonological processing for speech perception and production. Cognitive Science, 25, 663-678.

Burgess, P. W., \& Shallice, T. (1997). The Hayling and Brixton tests. Bury St. Edmunds Thames Valley Test Company.

Cabeza, R., \& Nyberg, L. (2000). Imaging cognition II: An empirical review of 275 PET and fMRI studies. Journal of Cognitive Neuroscience, 12, 1-47.

Chertkow, H., Bub, D., Deaudon, C., \& Whitehead, V. (1997). On the status of object concepts in aphasia. Brain and Language, 58, 203-232.

Cohen, R., Kelter, S., \& Woll, G. (1980). Analytical competence and language impairment in aphasia. Brain and Language, 10, 331-351.

Corbett, F., Jefferies, E., Ehsan, S., \& Ralph, M. A. L. (2009). Different impairments of semantic cognition in semantic dementia and semantic aphasia: Evidence from the non-verbal domain. Brain, 132, 2593-2608.

Damasio, H., \& Damasio, A. R. (1989). Lesion analysis in neuropsuchology. New York: Oxford University Press.

Davis, C. J. (2005). N-Watch: A program for deriving neighborhood size and other psycholinguistic statistics. Behavior Research Methods, 37(1), 65-70.

De Renzi, E., Faglioni, P., Scotti, G., \& Spinnler, H. (1972). Impairment in associating colour to form, concomitant with aphasia. Brain, 95(2), 293-304.

Dronkers, N. F., Redfern, B. B., \& Ludy, C. A. (1995). Lesion localization in chronic Wernicke's aphasia. Brain and Language, 51, 62-65.

Dronkers, N. F., Wilkins, D. P., Van Valin, R. D., Jr., Redfern, B. B., \& Jaeger, J. J. (2004). Lesion analysis of the brain areas involved in language comprehension. Cognition, 92, 145-177.

Eggert, G. H. (1977). Wernicke's works on aphasia: A sourcebook and review. The Hague: Mouton Publishers.

Ellis, A. W., Miller, D., \& Sin, G. (1983). Wernicke's aphasia and normal language processing: A case study in cognitive neuropsychology. Cognition, 15, 111-144.

Gainotti, G., \& Lemmo, M. S. (1976). Comprehension of symbolic gestures in aphasia. Brain and Language, 3, 451-460.

Gainotti, G., Silveri, M. C., Villa, G., \& Caltagirone, C. (1983). Drawing objects from memory in aphasia. Brain, 106, 613-622.

Garrard, P., Lambon Ralph, M. A., Hodges, J. R., \& Patterson, K. (2001). Prototypicality, distinctiveness, and intercorrelation: Analyses of the semantic attributes of living and nonliving concepts. Cognitive Neuropsychology, 18, 125-174. 
Glasser, M. F., \& Rilling, J. K. (2008). DTI tractography of the human brain's language pathways. Cerebral Cortex, 18, 2471-2482.

Goodglass, H., Kaplan, E., \& Barresi, B. (2001a). The assessment of aphasia and related disorders (3rd ed.). Baltimore: Lippincott Williams \&Wilkins.

Goodglass, H., Kaplan, E., \& Barresi, B. (2001b). Boston Diagnostic Aphasia Examination (3rd ed.). Baltimore: Lippincott Williams \& Wilkins. (BDAE).

Grant, D. A., \& Berg, E. A. (1993). The Wisconsin Card Sort task. San Antonio, TX: Psychological Assessment Resources, Inc.

Graves, W. W., Grabowski, T. J., Mehta, S., \& Gupta, P. (2008). The left posterior superior temporal gyrus participates specifically in accessing lexical phonology. Journal of Cognitive Neuroscience, 20, 1698-1710.

Head, H. (1926). Aphasia and kindred disorders of speech. London: CUP.

Heim, S., \& Friederici, A. D. (2003). Phonological processing in language production: Time course of brain activity. Neuroreport, 14, 2031-2033.

Hickok, G. (2000). Speech perception conduction aphasia and the functional neuroanatomy of language. In Y. Grodzinsky, L. P. Shapiro, \& D. Swinney (Eds.), Language and the brain: Representation and processing. San Diego: Academic Press.

Hoffman, P., Pobric, G., Drakesmith, M., \& Lambon Ralph, M. A. (in press). Posterior middle temporal gyrus is necessary for verbal and non-verbal semantic cognition: Evidence from rTMS. Aphasiology, doi:10.1080/02687038.2011.608838.

Howard, D., \& Patterson, K. (1992). Pyramids and palm trees: A test of semantic access from pictures and words. Bury Saint Edmunds: Thames Valley Test Company.

Indefrey, P., \& Levelt, W. J. M. (2004). The spatial and temporal signatures of word production components. Cognition, 92, 101-144.

Jefferies, E., \& Lambon Ralph, M. A. (2006). Semantic impairment in stroke aphasia versus semantic dementia: A case-series comparison. Brain, 129(8), 2132-2147.

Kay, J., Coltheart, M., \& Lesser, R. (1992). Psycholinguistic assessments of language processing in aphasia. Hove, UK: Laurence Erlbaum Associates. (PALPA)

Lambon Ralph, M. A., Lowe, C., \& Rogers, T. T. (2007). Neural basis of category-specific semantic deficits for living things: Evidence from semantic dementia, HSVE and a neural network model. Brain, 130, 1127-1137.

Lambon Ralph, M. A., Sage, K., Jones, R. W., \& Mayberry, E.J. (2010). Coherent concepts are computed in the anterior temporal lobes. Proceedings of the National Academy of Sciences of the United States of America, 107, 2717-2722.

Leff, A. P., Schofield, T. M., Crinion, J. T., Seghier, M. L., Grogan, A., Green, D. W., et al. (2009). The left superior temporal gyrus is a shared substrate for auditory short-term memory and speech comprehension: Evidence from 210 patients with stroke. Brain, 132, 3401-3410.

Lichtheim, L. (1885). On aphasia. Brain, 7, 433-484.

Luria, A. R. (1976). Disturbances of understanding of verbal communication in patients with sensory aphasia. The Hague: Mouton \& Co. B.V.

Luria, A. R., \& Hutton, J. T. (1977). A modern assessment of the basic forms of aphasia. Brain and Language, 4, 129-151.

Lytton, W. W., \& Brust, J. C. M. (1989). Direct dyslexia. Brain, 112, 583-594.

Morris, J., Franklin, S., Ellis, A. W., Turner, J. E., \& Bailey, P. J. (1996). Remediating a speech perception deficit in an aphasic patient. Aphasiology, 10(2), 137-158.

Mummery, C. J., Ashburner, J., Scott, S. K., \& Wise, R. J. S. (1999). Functional neuroimaging of speech perception in six normal and two aphasic subjects. Journal of the Acoustical Society of America, 106, 449-457.

Narain, C., Scott, S. K., Wise, R. J. S., Rosen, S., Leff, A. P., Iversen, S. D., et al. (2003). Defining a left-lateralized response specific to intelligible speech using fMRI. Cerebral Cortex, 13, 1362-1368.

Nestor, P. J., Fryer, T. D., \& Hodges, J. R. (2006). Declarative memory impairments in Alzheimer's disease and semantic dementia. Neurolmage, 30(3), 1010-1020.

Noonan, K. A., Jefferies, E., Corbett, F., \& Lambon Ralph, M. A. (2010). Elucidating the nature of deregulated semantic cognition in semantic aphasia: Evidence for the roles of prefrontal and temporoparietal cortices. Journal of Cognitive Neuroscience, 22, 1597-1613.
Ogar, J. M., Baldo, J. V., Wilson, S. M., Brambati, S. M., Miller, B. L., Dronkers, N. F., et al. (2011). Semantic dementia and persisting Wernicke's aphasia: Linguistic and anatomical profiles. Brain and Language, 117(1), 28-33.

Patterson, K., Nestor, P. J., \& Rogers, T. T. (2007). Where do you know what you know? The representation of semantic knowledge in the human brain. Nature Reviews. Neuroscience, 8, 976.

Pobric, G., Jefferies, E., \& Lambon Ralph, M. A. (2010a). Amodal semantic representations depend on both anterior temporal lobes: Evidence from repetitive transcranial magnetic stimulation. Neuropsychologia, 48, 1336-1342.

Pobric, G., Jefferies, E., \& Lambon Ralph, M. A. (2010b). Category-specific versus category-general semantic impairment induced by transcranial magnetic stimulation. Current Biology, 20(10), 964-968.

Raven, J. C. (1962). Coloured Progressive Matrices sets A, AB, B. London: H. K. Lewis.

Robertson, I. H., Ward, T., Ridgeway, V., \& Nimmo-Smith, I. (1994). The Test of Everyday Attention. London: Thames Valley Test Company.

Robson, H., Lambon Ralph, M. A., \& Sage, K. (in press). Revealing and quantifying the impaired phonological analysis underpinning impaired comprehension in Wernicke's aphasia. Neuropsychologia, doi:10.1016/j.neuropsychologia.2011.11.022.

Rogers, T. T., Lambon Ralph, M. A., Garrard, P., Bozeat, S., McClelland, J. L., Hodges, J. R., et al. (2004). Structure and deterioration of semantic memory: A neuropsychological and computational investigation. Psychological Review, 111(1), 205-235.

Rorden, C. (2005). MRIcro: http://www.sph.sc.edu/comd/rorden/mricro.html

Saur, D., Kreher, B., Schnell, S., Kummerer, D., Kellmeyer, P., \& Vry, M. (2008). Ventral and dorsal pathways for language. Proceedings of the National Academy of Sciences of the United States of America, 105, 18035-18040.

Saur, D., Schelter, B., Schnell, S., Kratochvil, D., Küpper, H., Kellmeyer, P., et al. (2010). Combining functional and anatomical connectivity reveals brain networks for auditory language comprehension. NeuroImage, 49(4), 3187-3197.

Seghier, M. L., Ramlackhansingh, A., Crinion, J., Leff, A. P., \& Price, C. J. (2008). Lesion identification using unified segmentation-normalisation models and fuzzy clustering. Neurolmage, 41(4), 1253-1266.

Thompson-Schill, S. L., D’Esposito, M., Aguirre, G. K., \& Farah, M. J. (1997). Role of left inferior prefrontal cortex in retrieval of semantic knowledge: A reevaluation. Proceedings of the National Academy of Sciences of the United States of America, 94, $14792-14797$.

Vigneau, M., Beaucousin, V., Herve, P. Y., Duffau, H., Crivello, F., Houde, O., et al. (2006). Meta-analyzing left hemisphere language areas: Phonology, semantics, and sentence processing. NeuroImage, 30, 1414-1432.

Visser, M., Jefferies, E., \& Ralph, M. A. L. (2010). Semantic processing in the anterior temporal lobes: A meta-analysis of the functional neuroimaging literature. Journal of Cognitive Neuroscience, 22(6), 1083-1094

Wagner, A. D., Pare-Blagoev, E. J., Clark, J., \& Poldrack, R. A. (2001). Recovering meaning: Left prefrontal cortex guides controlled semantic retrieval. Neuron, 31, 329-338.

Warren, J. E., Wise, R. J. S., \& Warren, J. D. (2005). Sounds do-able: Auditory-motor transformations and the posterior temporal plane. Trends in Neurosciences, 28(12), 636-642.

Warrington, E. K., \& James, M. (1991). The visual object and space perception battery. Bury St. Edmunds, UK: Thames Valley Test Company.

Warrington, E. K., \& Leff, A. P. (2000). Jargon dyslexia: A single case study of intact reading comprehension in a jargon dysphasic. Neurocase, 6, 499-507.

Whitney, C. M., Kirk, M., O'Sullivan, J., Lambon Ralph, M. A., \& Jefferies, E. (2011). The neural organization of semantic control: TMS evidence for a distributed network in left inferior frontal and posterior middle temporal gyrus. Cerebral Cortex, 21, 1066-1075.

Wise, R. J. S., Scott, S. K., Blank, C., Mummery, C. J., Murphy, K., \& Warburton, E. A. (2001). Separate neural subsystems within 'Wernicke's area'. Brain, 124, 83-95. 